\title{
Epidemic Use of Benzodiazepines among Older Adults in Israel: Epidemiology and Leverage Points for Improvement
}

\author{
Michael A. Steinman, MD 1,2,3, Marcelo Low, MPH',3, Ran D. Balicer, MD, PhD 2,4, and Efrat Shadmi, \\ $R N, P h D^{2,3}$
}

'University of California, San Francisco, and the San Francisco VA Health Care System, San Francisco, CA, USA; ${ }^{2}$ Clalit Research Institute, Tel Aviv, Israel; ${ }^{3}$ University of Haifa, Haifa, Israel; ${ }^{4}$ Ben Gurion University of the Negev, Beersheva, Israel.

BACKGROUND: Benzodiazepines and benzodiazepinereceptor agonists (BDZRAs, often known as " $Z$-drugs") are commonly used in older adults despite welldocumented harms.

OBJECTIVE: To evaluate patterns of benzodiazepine and BDZRA use in Israel, focusing on potential leverage points where quality improvement initiatives might effectively curtail new use or the transition from intermittent to chronic use.

DESIGN AND PARTICIPANTS: We used national electronic medical data to assess a $10 \%$ random sample of adults receiving care in Clalit Health Services, which serves half of Israel's population. The sample included 267,221 adults, of whom 56,808 (21\%) were age 65 and older.

MAIN MEASURES: Medication use from 2013 to 2015 was ascertained using pharmacy dispensing data.

RESULTS: In 2014, 7\% of adults age 21-64 and 32\% of adults age 65 and older received at least one benzodiazepine/BDZRA, including 49\% of adults age 85 and older $(P<0.001)$. The majority of older users $(59 \%)$ were longterm users of the drugs, and $21 \%$ of older adults who were short-term users in 2014 transitioned to medium- or long-term use in 2015. Older Arab Israelis were much less likely to receive benzodiazepine/BDZRAs than older Jewish Israelis (adjusted OR 0.28, 95\% 0.25-0.31), but within each community there was no major variation in prescribing rates across clinics. Depression diagnosis was associated with particularly high rates of benzodiazepine/ BDZRA use: $17 \%$ of older adults with depression received a benzodiazepine/BDZRA but no antidepressant, and $42 \%$ received both. Recent hospitalization increased the risk of new benzodiazepine/BDZRA use (adjusted OR $1.41,95 \%$ CI 1.01-1.96), but the absolute risk increase was only $3 \%$.

CONCLUSIONS: Benzodiazepines/BDZRAs are used at exceptionally high rates by older Israeli adults, especially the oldest old. Important leverage points for quality improvement efforts include curtailing the transition from short-term to long-term use, reducing use in older adults with depression, and identifying reasons that explain

Electronic supplementary material The online version of this article (doi:10.1007/s11606-017-4059-1) contains supplementary material, which is available to authorized users.

Received December 8, 2016

Revised March 13, 2017

Accepted March 31, 2017

Published online May 3, 2017 large differences in benzodiazepine/BDZRA prescribing between different ethnic groups.

KEY WORDS: aging; ambulatory care; health services research; pharmaceutical care; pharmacoepidemiology.

J Gen Intern Med 32(8):891-9

DOI: $10.1007 / \mathrm{s} 11606-017-4059-1$

(C) Society of General Internal Medicine 2017

\section{INTRODUCTION}

Benzodiazepines are commonly prescribed in many countries, including Israel. ${ }^{1-8}$ This is problematic because these drugs can cause substantial harm. In older adults, benzodiazepine use increases the risk of falls, fracture, cognitive impairment, motor vehicle accidents, and other adverse outcomes. ${ }^{9-11}$ Similar risks have been found with benzodiazepine-like agents such as zolpidem and zopiclone, collectively known as benzodiazepine-receptor agonists (BDZRAs). ${ }^{9}, 12,13$

Previous studies have documented the frequency of and risk factors for benzodiazepine and BDZRA use in different settings. ${ }^{1-8,}{ }^{14}$ However, much less is known about potential leverage points that can be targeted to reduce high-risk use. The best opportunities may lie in curtailing the initiation of these drugs and preventing the transition from acute or intermittent use to chronic use, as it is very difficult to discontinue benzodiazepines in patients who have been using them chronically, even with comprehensive interventions. ${ }^{15,}{ }^{16}$ In order to design the next generation of strategies for attacking the problem upstream, an understanding of this phase of the medication use process is critical. This includes knowledge of events that might trigger initiation of benzodiazepines and BDZRAs, characteristics of the transition from short-term to long-term use, and the extent to which population-wide use is attributable to a few high-prescribing physicians versus a broad-based phenomenon.

In this study, we used national data from Israel's largest health care provider to help answer these questions. Our initial analyses focused on evaluating patterns of benzodiazepine and BDZRA use in the general adult population. Additional, indepth analyses of potential leverage points focused on older adults, since they are the most frequent users of these drugs and have a high risk of adverse effects. 


\section{METHODS}

\section{Subjects and Data Sources}

Our study used a random $10 \%$ sample of adults age 21 years and older who were enrolled in Clalit Health Services in 2015. Clalit is a not-for-profit integrated care delivery system that serves approximately one-half of Israel's population. We excluded subjects who could not be linked to a primary care physician in 2014 or 2015 (3.5\% of the sample). All data for this project were obtained from Clalit clinical and administrative data systems.

\section{Measures}

Medications. Medication use was assessed using dispensing records from Clalit pharmacies and from private pharmacies that fill prescriptions ordered by Clalit providers. Together, these account for the vast majority of prescription medications dispensed to Clalit patients. We combined benzodiazepines and BDZRAs into a single category, because both drug types carry similar risks for adverse events in older adults, including daytime drowsiness, cognitive impairment, and falls. ${ }^{9}, 11$

In Clalit, benzodiazepines/BDZRAs are typically prescribed for a 1-month supply: in our sample, $83 \%$ of dispensings (also known as "fills") were for a 28- to 30-day supply, $12 \%$ were for less than 28 days, and $5 \%$ were for more than 30 days. We thus counted the number of dispensings in each calendar year as a marker of duration of benzodiazepine/ BDZRA use. Based on previous studies, we categorized patients as "short-term users" (1-2 dispensings during a calendar year), "medium-term users" (3-5 dispensings), or "long-term users" (6 or more dispensings). ${ }^{17}$

We used a pharmacy reference source (Micromedex) to identify the lowest daily maintenance dose recommended for each benzodiazepine and BDZRA; we defined these doses as low-dose therapy (Online Appendix 1). ${ }^{18}$

Diagnoses. We evaluated diagnoses associated with specific visits in 2014 as well as chronic diagnoses entered by physicians between 2010 and 2014. Mental health care in Clalit is a carve-out program, and diagnoses from mental health visits were not available. We also used a chronic conditions registry maintained by Clalit, which identifies diagnoses based on a variety of data sources. For diagnoses potentially related to benzodiazepine or BDZRA use (e.g., anxiety, depression), we considered a diagnosis from any of these sources as indicating the presence of that condition. For contextual diagnoses such as diabetes, we used only the chronic conditions registry. Diagnoses of sleep disorders were not available in the data.

Other Variables. Ethnic/religious groups were classified by subjects' birth country and the predominant population served by clinics in which those patients received care (e.g., Jewish or Arab). Although the latter is a clinic-level variable, given the highly segregated nature of Israeli society, it is considered a good proxy for patient ethnicity. ${ }^{1}$ Since nearly all Israeli Arabs were born in Israel and its environs, we did not create a separate category for Arab immigrants. Low socioeconomic status was identified by the presence of pharmacy subsidies given to patients with low economic means. ${ }^{19}$ The burden of multimorbidity was estimated with the Adjusted Clinical Groups $^{\circledR}\left(\mathrm{ACG}^{\circledR}\right)$ case-mix system. ${ }^{20}$

\section{Analyses}

We used the chi-square test for bivariate comparisons and multivariable logistic regression to evaluate independent predictors of benzodiazepine/BDZRA use. To study the impact of hospitalization on initiation of benzodiazepine/BDZRAs, we compared rates of use in older adults who were hospitalized in 2014 and those who were not, using multivariable logistic regression to adjust for differences between groups (Online Appendix 2). For analyses of clinic-level variation in benzodiazepine/BDZRA use, we adjusted clinic-level prescribing rates for the distribution of age, sex, and ACG scores of patients cared for in that clinic. These analyses included only clinics with 100 or more older patients in our data set, so as to generate reliable clinic-level estimates, and excluded physicians who were independent contractors with Clalit, as clinic-level prescribing rates could not be reliably ascertained for these practices. For analyses of concurrent use of multiple agents, we evaluated medications actively in use on 1 January 2015 per the methods described by Lund et al. ${ }^{21}$

This research was approved by the Institutional Review Board/Helsinki Committee of Clalit Health Services and the University of California, San Francisco.

\section{RESULTS}

Characteristics of subjects are shown in Table 1. Among 56,808 patients age 65 and older, $57 \%$ were women, and $41 \%$ were of low socioeconomic status.

\section{Use of Benzodiazepines and Benzodiazepine- Receptor Agonists (BDZRAs)}

Prevalence of Use. During 2014, $12 \%$ of adults had at least one dispensing of a benzodiazepine/BDZRA. This included $7 \%$ of adults age $21-64$ and $32 \%$ of adults age 65 and older, including $49 \%$ of adults age 85 and older (Fig. 1). At a single point in time (1 January 2015), 17\% of benzodiazepine/ BDZRA users had a concurrent supply of two or more different types of benzodiazepines or BDZRAs.

Type of Agents Used. Among older adults who used either a benzodiazepine or BDZRA as of 1 January 2015, 79\% were using only a benzodiazepine, $14 \%$ were using only a BDZRA, 
Table 1 Characteristics of Cohort, by Age Group

\begin{tabular}{|c|c|c|}
\hline & $\begin{array}{l}\text { Age } 21-64 \\
\text { years } \\
\text { no. }(\%) \\
(N=210,413)\end{array}$ & $\begin{array}{l}\text { Age } 65 \text { years and } \\
\text { older } \\
\text { no. }(\%) \\
(N=56,808)\end{array}$ \\
\hline \multicolumn{3}{|l|}{ Age (years): } \\
\hline $21-44$ & $131,969(63 \%)$ & - \\
\hline $45-54$ & $38,126(18 \%)$ & - \\
\hline $55-64$ & $40,318(19 \%)$ & - \\
\hline $65-74$ & - & $29,083(51 \%)$ \\
\hline $75-84$ & - & $18,940(33 \%)$ \\
\hline $85+$ & - & $8785(15 \%)$ \\
\hline Female sex & $107,677(51 \%)$ & $32,423(57 \%)$ \\
\hline \multicolumn{3}{|l|}{ Ethnicity/religion* } \\
\hline Jewish, born in Israel & $117,905(56 \%)$ & $11,269(20 \%)$ \\
\hline Jewish, born in Russia or & $20,070(10 \%)$ & $16,767(30 \%)$ \\
\hline \multicolumn{3}{|l|}{ E. Europe } \\
\hline Jewish, born in Middle & $6865(3 \%)$ & $18,479(33 \%)$ \\
\hline \multicolumn{3}{|l|}{ East or N. Africa } \\
\hline $\begin{array}{l}\text { Jewish, born elsewhere or } \\
\text { unknown }\end{array}$ & $12,613(6 \%)$ & $4996(9 \%)$ \\
\hline Arab & $52,960(25 \%)$ & $5297(9 \%)$ \\
\hline Low socioeconomic status & $21,564(10 \%)$ & $23,074(41 \%)$ \\
\hline \multicolumn{3}{|l|}{ ACG score } \\
\hline 1-2 (most healthy) & $80,376(38 \%)$ & $6138(11 \%)$ \\
\hline $3-4$ & $117,742(56 \%)$ & $32,119(57 \%)$ \\
\hline 5-6 (least healthy) & $12,295(6 \%)$ & $18,551(33 \%)$ \\
\hline \multicolumn{3}{|l|}{ Chronic medical conditions } \\
\hline Hypertension & $24,030(11 \%)$ & $38,251(67 \%)$ \\
\hline Diabetes & $16,382(8 \%)$ & $19,437(34 \%)$ \\
\hline Ischemic heart disease & $6533(3 \%)$ & $15,420(27 \%)$ \\
\hline \multicolumn{3}{|c|}{ Benzodiazepine-related health conditions } \\
\hline Depression & $10,615(5 \%)$ & $8913(16 \%)$ \\
\hline Anxiety & $8247(4 \%)$ & $5620(10 \%)$ \\
\hline Adjustment disorder & $530(0.3 \%)$ & $268(0.5 \%)$ \\
\hline Dementia & $326(0.2 \%)$ & $5059(9 \%)$ \\
\hline Epilepsy & $2631(1 \%)$ & $1029(2 \%)$ \\
\hline \multicolumn{3}{|c|}{ Number of medication classes used during 2014} \\
\hline 0 & $45,114(21 \%)$ & $1777(3 \%)$ \\
\hline $1-4$ & $98,200(47 \%)$ & $7736(14 \%)$ \\
\hline $5-9$ & $46,983(22 \%)$ & $17,833(31 \%)$ \\
\hline$\geq 10$ & $20,116(10 \%)$ & $29,462(52 \%)$ \\
\hline \multicolumn{3}{|c|}{ Medications used at any point during 2014} \\
\hline Antidepressant & $14,427(7 \%)$ & $11,768(21 \%)$ \\
\hline Opioid analgesic & $10,737(5 \%)$ & $8763(15 \%)$ \\
\hline Anti-dementia medication & $51(<0.1 \%)$ & $1513(3 \%)$ \\
\hline \multicolumn{3}{|c|}{ No. of hospitalizations during 2014} \\
\hline 0 & $193,859(92 \%)$ & $45,089(79 \%)$ \\
\hline 1 & $12,774(6 \%)$ & $7829(14 \%)$ \\
\hline 2 or more & $3780(2 \%)$ & $3890(7 \%)$ \\
\hline \multicolumn{3}{|c|}{ No. of primary care physician visits during 2014} \\
\hline 0 & $30,353(14 \%)$ & $4634(8 \%)$ \\
\hline $1-4$ & $97,600(46 \%)$ & $15,992(28 \%)$ \\
\hline $5-9$ & $53,155(25 \%)$ & $17,348(31 \%)$ \\
\hline$\geq 10$ & $29,305(14 \%)$ & $18,834(33 \%)$ \\
\hline
\end{tabular}

and $6 \%$ had concurrent dispensings for both. Results were almost identical for younger adults. Clonazepam was the most commonly dispensed agent for younger adults $(28 \%)$, whereas in adults age 65 and older, brotizolam was most commonly used (46\%; Online Appendix 3).

Duration of Use. Among individuals who received at least one benzodiazepine/BDZRA in 2014, 33\% of adults age 2164 were long-term users ( 6 or more dispensings). In contrast, $59 \%$ of users age 65 and older were long-term users, including $72 \%$ of adults age 85 and older $(P<0.001$; Fig. 1 and Online Appendix 4). On a population level, $2 \%$ of adults age $21-64$ and $19 \%$ of adults age 65 and older were long-term users of benzodiazepines in $2014(P<0.001)$.

Dose. Overall, 57\% of users age 21-64 received low-dose prescriptions, compared with $74 \%$ among adults age 65 and older $(P<0.001)$.

\section{Characteristics Associated with Benzodiazepine/BDZRA}

Use in Older Adults. The strongest independent predictors of any benzodiazepine/BDZRA use in older adults included age, female sex, religious/ethnic group, depression, anxiety, and use of an antidepressant (Table 2). Compared with Jewish Israelis (all subgroups combined), Arab Israelis had substantially lower odds of receiving a benzodiazepine/BDZRA (adjusted OR $0.28,95 \%$ CI $0.25-0.31$ ). Similar factors were associated with long-term use (Online Appendix 5).

\section{Problem Areas and Potential Leverage Points for Quality Improvement}

We next investigated several areas that we hypothesized could have special implications for quality improvement. These included characterizing the transition from short-term to longer-term use, hospitalization as a trigger for new use, identifying whether benzodiazepine/BDZRA prescribing was concentrated in a limited number of clinics or was a broadbased phenomenon, and depression as a high-risk diagnosis for use.

Initiation of Use, Persistence of Use, and Transition from Short-Term to Longer-Term Use. Among older adults with no benzodiazepine/BDZRA use in 2014, 10\% received at least one of these medications in 2015. Most of these new users were short-term users of the medication (1-2 dispensings per year; Fig. 2). Among older adults with short-term benzodiazepine/BDZRA use in 2014, 21\% transitioned to higher rates of use in the following year, including $12 \%$ who transitioned to medium-term use (3-5 dispensings) and 9\% who transitioned to long-term use (6 or more dispensings). The vast majority ( $87 \%$ ) of patients who were long-term users in 2014 remained long-term users in 2015.

Impact of Hospitalization on Initiation of Use. Among 6834 older adults who were non-users of benzodiazepine/BDZRAs for at least 1 year prior to hospitalization, $5 \%$ initiated the drug within 2 months after hospitalization. This compares to a background rate of initiation of $2 \%$ among 32,141 older nonusers who were not hospitalized $(P<0.001$; Online Appendix 6). After adjusting for baseline subject characteristics, hospitalization was associated with an increased risk of starting benzodiazepines/BDZRAs (OR 1.41, 95\% CI 1.01-1.96).

Most of the $5 \%$ of older adults who were newly prescribed a benzodiazepine/BDZRA within 2 months of hospitalization did not become regular users: $69 \%$ had a total of only one or 


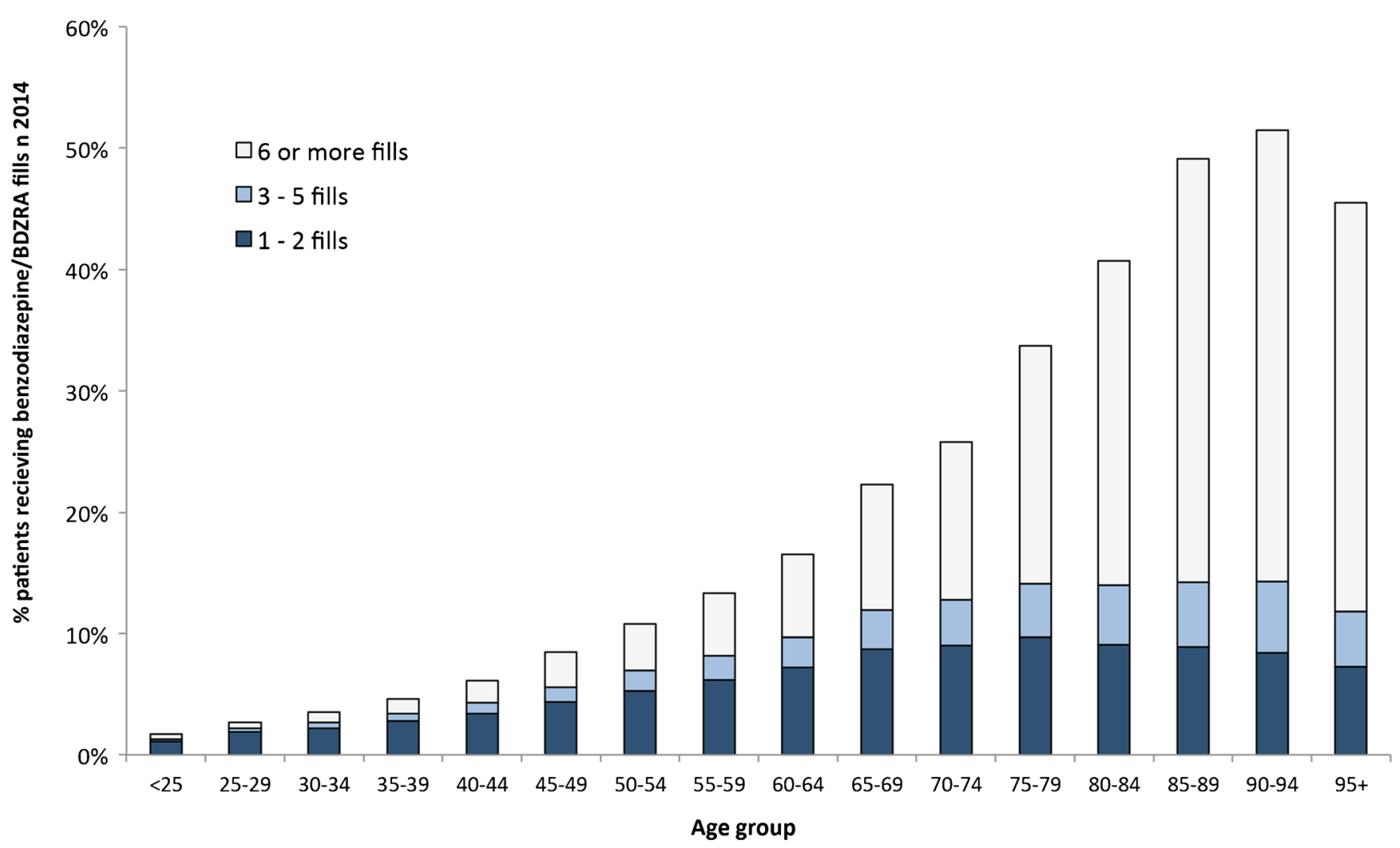

Figure 1 Use of benzodiazepine/BDZRAs during 2014, by age. The total height of each bar shows the percentage of people in that age group who received at least one dispensing of a benzodiazepine/BDZRA during 2014. In each age group, use is subdivided into people who received 12 dispensings during 2014 ("short-term users"), people who received 3-5 dispensings ("medium-term users"), and those who received 6 or more dispensings ("long-term users").

two dispensings of the drug in the first 6 months after hospitalization. However, $43 \%$ of older adults who received a benzodiazepine/BDZRA after hospitalization were still using the drug between 12 and 18 months later. In contrast, $10 \%$ of adults who were hospitalized but did not start the drug in the post-hospital period had at least one dispensing 12-18 months later (adjusted OR 3.01, 95\% CI 2.22-4.08).

Among individuals with prior but not recent benzodiazepine/BDZRA use, hospitalization was associated with a $6 \%$ absolute increase in the risk of re-initiating benzodiazepine/BDZRA use (19\% of hospitalized older adults vs. a background rate of $13 \%$ in non-hospitalized older adults, $P<0.001)$. The difference was reduced after adjusting for subject characteristics (adjusted OR 1.27, 95\% CI, 0.84-1.92; Online Appendix 6).

Variation in Use Across Primary Care Clinics. Figure 3 shows the percentage of adults age 65 years and older within each primary care clinic who received a benzodiazepine/ BDZRA in 2014, adjusted for the age, sex, and ACG score of patients within that clinic. The population-adjusted median rate of prescribing was 36\%. Clinics in the 10th and 90th percentiles of prescribing had rates of $26 \%$ and $43 \%$, respectively. There were only two clinics serving a predominantly Arab population that met inclusion criteria for this analysis.
Their population-adjusted prescribing rates were $12 \%$ and $14 \%$.

Relationship with Depression Diagnosis and Use of Antidepressants. Sixteen percent of older adults $(N=8913)$ had a diagnosis of depression. Among these individuals, 20\% received an antidepressant and no benzodiazepine/BDZRA in 2014, 17\% received a benzodiazepine/BDZRA and no antidepressant, $42 \%$ received both types of agents, and $21 \%$ received neither (Online Appendix 7). Benzodiazepine/ BDZRA users with a diagnosis of depression were more likely to be long-term users than benzodiazepine/BDZRA users without depression $(69 \%$ vs. $55 \%, P<.001$; adjusted OR $1.44,95 \% 1.34-1.55)$.

\section{DISCUSSION}

In this study from Israel's largest health care provider, the use of benzodiazepine and benzodiazepine-receptor agonists (BDZRAs) was extraordinarily high in older adults, particularly the oldest old. Nearly half of adults age 85 years and older received a benzodiazepine or BDZRA in 2014, with nearly three-quarters of these being long-term users. There 
Table 2 Characteristics Associated with Benzodiazepine/BDZRA Use among Adults Age 65 and Older, 2014

\begin{tabular}{|c|c|c|c|c|}
\hline & \multirow[b]{3}{*}{ No. in group } & \multicolumn{3}{|c|}{ Any benzodiazepine/BDZRA use } \\
\hline & & \multicolumn{2}{|c|}{ Bivariate analyses } & \multirow{2}{*}{$\frac{\text { Multivariable analyses }}{\text { Adjusted odds ratio }(95 \% \mathrm{CI})^{*}}$} \\
\hline & & Percentage & $P$ value & \\
\hline \multicolumn{5}{|l|}{ Age (years) } \\
\hline $65-74$ & 29,083 & $24 \%$ & \multirow[t]{3}{*}{$<0.001$} & - \\
\hline $75-84$ & 18,940 & $37 \%$ & & $1.31(1.25-1.37)$ \\
\hline $85+$ & 8785 & $49 \%$ & & $1.86(1.75-1.98)$ \\
\hline \multicolumn{5}{|l|}{ Sex } \\
\hline Male & 24,385 & $25 \%$ & \multirow{2}{*}{$<0.001$} & - \\
\hline Female & 32,423 & $37 \%$ & & $1.41(1.35-1.47)$ \\
\hline \multicolumn{5}{|l|}{ Ethnicity/religion } \\
\hline Jewish, born in Israel & 11,269 & $30 \%$ & \multirow[t]{5}{*}{$<0.001$} & - \\
\hline Jewish, born in Russia or E. Europe & 16,767 & $43 \%$ & & $1.17(1.10-1.24)$ \\
\hline Jewish, born in Middle East or N. Africa & 18,479 & $29 \%$ & & $0.73(0.69-0.78)$ \\
\hline Jewish, born elsewhere or unknown & 4996 & $30 \%$ & & $0.88(0.81-0.95)$ \\
\hline Arab & 5297 & $12 \%$ & & $0.26(0.24-0.29)$ \\
\hline \multicolumn{5}{|l|}{ Socioeconomic status } \\
\hline Not low & 33,734 & $28 \%$ & \multirow[t]{2}{*}{$<0.001$} & - \\
\hline Low & 23,074 & $38 \%$ & & $1.10(1.05-1.15)$ \\
\hline \multicolumn{5}{|l|}{ ACG score (1-6) } \\
\hline 1-2 (most healthy) & 6138 & $18 \%$ & \multirow[t]{3}{*}{$<0.001$} & - \\
\hline $3-4$ & 32,119 & $29 \%$ & & $0.93(0.86-1.01)$ \\
\hline 5-6 (least healthy) & 18,551 & $42 \%$ & & $0.77(0.70-0.84)$ \\
\hline \multicolumn{5}{|l|}{ Chronic medical conditions* } \\
\hline Hypertension & 38,251 & $36 \%$ & $<0.001$ & $0.93(0.89-0.98)$ \\
\hline Diabetes & 19,437 & $32 \%$ & 0.10 & $0.66(0.63-0.69)$ \\
\hline Ischemic heart disease & 15,420 & $39 \%$ & $<0.001$ & $1.01(0.96-1.07)$ \\
\hline \multicolumn{5}{|l|}{ Benzodiazepine-related health conditions* } \\
\hline Depression & 8913 & $59 \%$ & $<0.001$ & $1.52(1.43-1.61)$ \\
\hline Anxiety & 5620 & $63 \%$ & $<0.001$ & $2.16(2.02-2.31)$ \\
\hline Adjustment disorder & 268 & $46 \%$ & $<0.001$ & $1.42(1.07-1.90)$ \\
\hline Dementia & 5059 & $50 \%$ & $<0.001$ & $1.19(1.09-1.29)$ \\
\hline Epilepsy & 1029 & $43 \%$ & $<0.001$ & $1.03(0.89-1.20)$ \\
\hline \multicolumn{5}{|l|}{ No. of medication classes used during year } \\
\hline 0 & 1777 & $0 \%$ & \multirow[t]{4}{*}{$<0.001$} & \multirow{4}{*}{$\begin{array}{l}1.15(1.15-1.16) \text { per } \\
\text { each additional } \\
\text { medication }\end{array}$} \\
\hline $1-4$ & 7736 & $7 \%$ & & \\
\hline $5-9$ & 17,833 & $22 \%$ & & \\
\hline$\geq 10$ & 29,462 & $47 \%$ & & \\
\hline \multicolumn{5}{|l|}{ Medications present at any point during year* } \\
\hline Antidepressant & 11,768 & $60 \%$ & $<0.001$ & $1.85(1.75-1.95)$ \\
\hline Opioid analgesic & 8763 & $46 \%$ & $<0.001$ & $0.97(0.91-1.02)$ \\
\hline Anti-dementia medication & 1513 & $51 \%$ & $<0.001$ & $0.73(0.63-0.83)$ \\
\hline \multicolumn{5}{|l|}{ No. of hospitalizations during year } \\
\hline 0 & 45,089 & $29 \%$ & $<0.001$ & - \\
\hline 1 & 7829 & $39 \%$ & & $0.95(0.89-1.01)$ \\
\hline 2 or more & 3890 & $48 \%$ & & $0.95(0.87-1.03)$ \\
\hline No. of primary care visits during year & & & & \\
\hline 0 & 4634 & $29 \%$ & $<0.001$ & - \\
\hline $1-4$ & 15,992 & $23 \%$ & & $0.85(0.77-0.93)$ \\
\hline $6-10$ & 17,348 & $30 \%$ & & $0.87(0.79-0.96)$ \\
\hline$>10$ & 18,834 & $42 \%$ & & $0.79(0.72-0.87)$ \\
\hline
\end{tabular}

Overall rate of benzodiazepine/BDZRA use among older adults in 2014 was $32 \%$, and overall rate of heavy use (6 or more dispensings) was $19 \%$ *Reference group is patients without each of these conditions or medications

were substantial differences in rates of prescribing between different sectors of Israeli society, with Jewish Israelis receiving benzodiazepines/BDZRAs far more frequently than Arab Israelis.

Several findings emerged regarding potential leverage points for quality improvement. A substantial minority of older adults who received short-term therapy in 2014 transitioned to medium- or long-term use in the following year, whereas nearly all subjects who were long-term users in 2014 remained at high levels of use. Older adults who were hospitalized were more likely to begin using benzodiazepines or BDZRAs than older adults who were not hospitalized, although the absolute risk increase was small (3\%). There was moderate variation in benzodiazepine/BDZRA prescribing rates among clinics, although rates of use were high in all but the lowest-prescribing clinics. Finally, benzodiazepine/ BDZRAs were used at very high rates in older adults with depression, often alongside or in place of antidepressant therapy.

Our results and those of another recent study suggest that the use of benzodiazepine/BDZRAs is substantially higher in Israel than in most other Western countries. ${ }^{22}$ In the United 


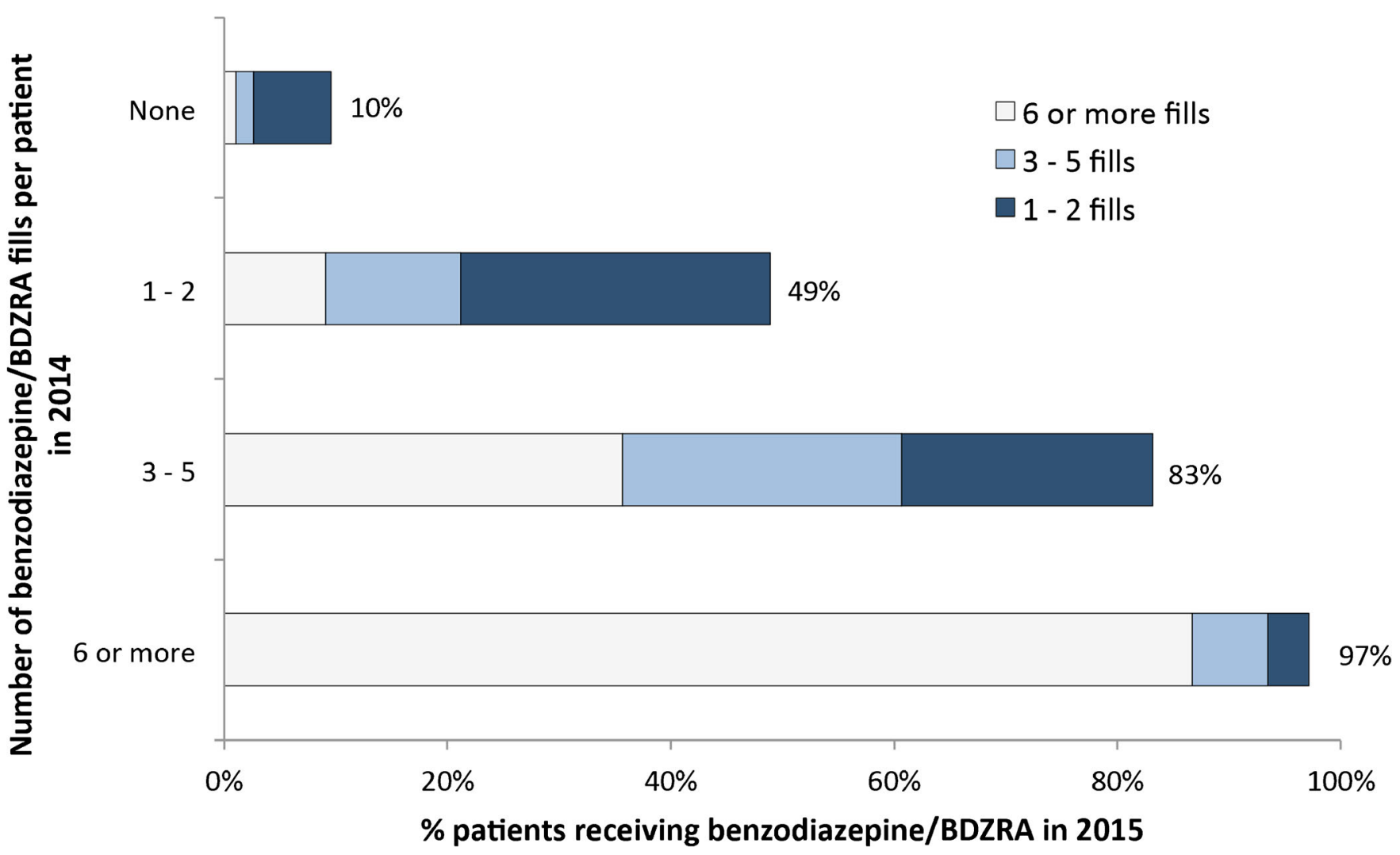

Figure 2 Use of benzodiazepines/BDZRAs by older adults in 2015, stratified by extent of use in the preceding year. Each horizontal bar shows the number of benzodiazepine/BDZRA dispensings in 2015. These bars are stratified by the extent of use in the previous year. For example, among older adults receiving no benzodiazepine/BDZRA dispensings in 2014, 10\% had at least one dispensing in 2015; most of these adults had only 1-2 dispensings in 2015.

States, approximately $9 \%$ of adults age $65-80$ years receive benzodiazepines at least once during a 1-year period, with one-third of these being long-term use. ${ }^{5,23}$ A recent study of European countries found period-prevalent use in roughly 6$18 \%$ of men and $11-37 \%$ of women age $60-69$ years, and in $12-30 \%$ of men and $17-45 \%$ of women age 80 years and older, with most countries on the lower end of this range. ${ }^{4}$ Similarly, a recent analysis of 13 member countries of the Organisation for Economic Co-operation and Development (OECD) found that Israel was in the highest tier of prescribing for long-term benzodiazepines. ${ }^{7}$

These results demonstrate an epidemic of benzodiazepine/ BDZRA use among older adults in Israel, with the most vulnerable group - the old-old - having the highest risk of use. Given the well-documented harms of these drugs in older adults, the pertinent question is how to reduce rates of benzodiazepine/BDZRA use. ${ }^{9-11}$ Because even the most intensive interventions have succeeded in discontinuing benzodiazepines in only one-quarter to one-third of older adults who are chronic users of these drugs, the most effective strategies may involve preventing initiation of benzodiazepines/BDZRAs in the first place, or intervening before individuals transition from short-term to longer-term use. ${ }^{15,16}$

Our results provide several insights into how this may be achieved. As shown in Figure 1, rates of short-term use remain fairly stable from age 60 and up; long-term use accounts for almost all of the dramatic increase in benzodiazepine/BDZRA use between the young-old and the old-old. Consistent with this finding, a substantial minority of older adults who were short-term users in one year progressed to medium- or long-term use in the following year (Fig. 2). Systems-based approaches may help blunt this transition from intermittent to chronic use. For example, electronic medical record-based alerts and pharmacy protocols could identify an accelerating pattern of prescriptions in people who were previously receiving these drugs only intermittently, and prompt intervention before a patient transitions to chronic and persistent use.

Due to limitations in the data used for this study, we could not reliably assess the reasons for which benzodiazepines and BDZRAs were prescribed. Because reasons for prescribing these medications (insomnia, anxiety) are often poorly documented and can overlap, other studies have faced similar difficulties. ${ }^{1,} 8,24$ Nonetheless, clinician education and decision support could be leveraged for the most common indications to reduce both short- and long-term use of these medications. For example, in the case of insomnia, such efforts could focus on improving diagnosis, supporting counseling on non-pharmacologic management strategies, and promoting the use of safer medications if drug therapy is indicated. ${ }^{25}$ 


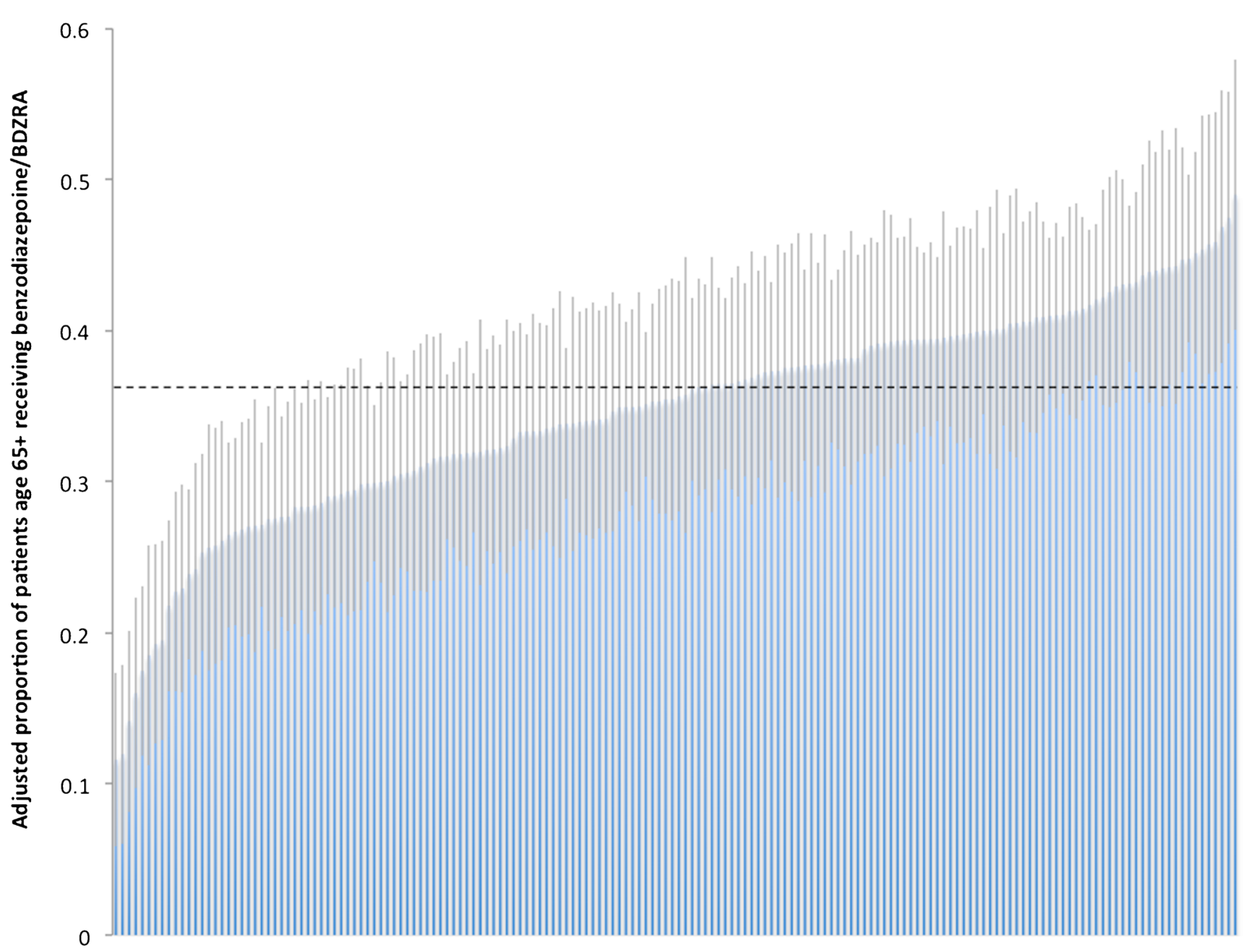

Figure 3 Percentage of people age 65 and older who received a benzodiazepine/BDZRA in 2014, by clinic. Results are adjusted for the distribution of age, sex, and ACG score of patients age 65 years and older within each clinic. Dashed bar shows the prescribing rate for the clinic at the 50th percentile of prescribing (36\%).

We hypothesized that hospitalization would be an important trigger for initiating benzodiazepine use. ${ }^{26-28}$ However, the absolute increase in benzodiazepine/ BDZRA initiation shortly following hospitalization was small (3\% over the background rate), and the population-attributable burden of hospitalization on benzodiazepine/BDZRA use is likely limited. Nonetheless, there may be a role for hospital-based initiatives, since the increased risk may compound over a period of years, and patients who are hospitalized are at high risk of adverse outcomes. Education and systems-based efforts could be effective in this setting, for example, with medication reconciliation systems flagging discharge prescriptions for a benzodiazepine/BDZRA among patients who were not chronic users prior to hospitalization.

Depression, which is common in Israeli elders, is another area where focus may be warranted. ${ }^{29}$ In these older adults,
benzodiazepines/BDZRAs were used as frequently as antidepressants, including as monotherapy in $17 \%$ of adults, and jointly with antidepressants in nearly half. Our results may underestimate the problem, since depression was likely undercoded in our data. Decision-support systems could prompt physicians to consider depression or dementia in older adults with insomnia or anxiety who might otherwise be prescribed benzodiazepines/BDZRAs, and help them target therapy to the underlying mental health condition. ${ }^{1,2}$ Recent reforms to better integrate mental health services into Israeli health plans have created a timely opportunity to develop and implement such programs.

We found little evidence of "hot-spot" primary care clinics that were responsible for a disproportionate amount of benzodiazepine/BDZRA prescribing. Together with prior research showing that physician characteristics have little impact on rates of benzodiazepine use, ${ }^{1}$ these results suggest that the problem will not be solved 
by targeting a limited number of high prescribers. However, the striking difference in rates of use between Arab and Jewish older adults points toward an opportunity. Emotional distress and mental health conditions are equally or more common in older Arab Israelis than in Jewish Israelis, yet rates of mental health treatment in the former are low due to cultural expectations, stigma, and norms of practice. ${ }^{30,31}$ Although this is problematic in many ways, in the case of benzodiazepines/BDZRAs, lower use probably represents better care, and could serve as an achievable benchmark to which to other communities can aspire. A better understanding of the reasons for differences in benzodiazepine/BDZRA prescribing between older Jewish and Arab populations could help inform strategies to reduce the use of these drugs for all Israelis.

Our study has several limitations. We did not have direct access to diagnoses from visits with mental health professionals, so our ability to capture a full range of psychiatric diagnoses was limited. Similarly, we lacked information on diagnoses of sleep disorders. We were unable to determine whether dispensed benzodiazepines/BDZRAs were used on a daily or as-needed basis, although this is unlikely to substantively affect our findings. Because of the sampling methods used, our analyses of clinic-level variation in benzodiazepine/ BDZRA prescribing was restricted to clinics caring for large numbers of older Clalit patients, and may not be representative of practices with smaller case loads.

High rates of benzodiazepine and BDZRA use among older Israelis pose a substantial threat to this population and merit an aggressive response. While efforts to reduce use of these drugs will be challenging, the magnitude of the problem calls for a multifaceted approach employing smart, targeted interventions.

Corresponding Author: Michael A. Steinman, MD; University of California, San Francisco, and the San Francisco VA Health Care System, San Francisco, CA, USA (e-mail: mike.steinman@ucsf.edu).

\section{Author Contributions}

- Study concept and design: Steinman, Low, Balicer, Shadmi.

- Data collection: Low, Balicer.

- Analysis and interpretation of data: Steinman, Low, Balicer, Shadmi.

- Drafting of initial version of the manuscript: Steinman.

- Review of manuscript for critical intellectual content: Low, Balicer, Shadmi.

\section{Compliance with Ethical Standards:}

Funding: Supported by the University of California San Francisco Research Allocation Program (Dr. Steinman).

Conflict of Interest: Dr. Steinman is a paid consultant for iodine.com. Mr. Low and Drs. Balicer and Shadmi declare that they do not have a conflict of interest.

Prior Presentations: None.

\section{REFERENCES}

1. Ayalon L, Gross R, Yaari A, Feldhamer E, Balicer RD, Goldfracht M. Patients' and physicians' characteristics associated with the purchase of benzodiazepines by older primary care patients in Israel. Admin Pol Ment Health 2013;40(2):117-123.

2. Ayalon L, Gross R, Yari A, Feldhamer E, Balicer RD, Goldfracht M. Factors associated with the type of psychotropic medications purchased for common mental disorders in the largest managed care organization in Israel. Int J Psychiatry Med 2012;44(1):91-102.

3. Blumstein T, Benyamini Y, Chetrit A, Mizrahi EH, Lerner-Geva L. Prevalence and correlates of psychotropic medication use among older adults in Israel: cross-sectional and longitudinal findings from two cohorts a decade apart. Aging Ment Health 2012;16(5):636-647.

4. Huerta C, Abbing-Karahagopian V, Requena G, et al. Exposure to benzodiazepines (anxiolytics, hypnotics and related drugs) in seven European electronic healthcare databases: a cross-national descriptive study from the PROTECT-EU Project. Pharmacoepidemiol Drug Saf 2016;25 Suppl 1:56-65.

5. Olfson M, King M, Schoenbaum M. Benzodiazepine use in the United States. JAMA Psychiatry 2015;72(2): 136-142.

6. Hollingworth SA, Siskind DJ. Anxiolytic, hypnotic and sedative medication use in Australia. Pharmacoepidemiol Drug Saf 2010; 19(3):280-288.

7. OECD. Prescribing in primary care. Health at a Glance 2015: OECD Indicators. Paris: OECD Publishing; 2015.

8. Maust DT, Kales HC, Wiechers IR, Blow FC, Olfson M. No end in sight: benzodiazepine use in older adults in the United States. J Am Geriatr Soc 2016.

9. Glass J, Lanctot KL, Herrmann N, Sproule BA, Busto UE. Sedative hypnotics in older people with insomnia: meta-analysis of risks and benefits. BMJ 2005;331(7526): 1169.

10. Petrov ME, Sawyer P, Kennedy R, Bradley LA, Allman RM. Benzodiazepine (BZD) use in community-dwelling older adults: longitudinal associations with mobility, functioning, and pain. Arch Gerontol Geriatr 2014;59(2):331-337.

11. American Geriatrics Society Beers Criteria Update Expert Panel. American Geriatrics Society 2015 updated beers criteria for potentially inappropriate medication use in older adults. J Am Geriatr Soc 2015;63(11):2227-2246.

12. Berry SD, Lee Y, Cai S, Dore DD. Nonbenzodiazepine sleep medication use and hip fractures in nursing home residents. JAMA Intern Med 2013;173(9):754-761.

13. Diem SJ, Ewing SK, Stone KL, et al. Use of non-benzodiazepine sedative hypnotics and risk of falls in older men. J Gerontol Geriatr Res 2014;3(3): 158.

14. Kroll DS, Nieva HR, Barsky AJ, Linder JA. Benzodiazepines are prescribed more frequently to patients already at risk for benzodiazepine-related adverse events in primary care. J Gen Intern Med 2016;31(9):1027-1034.

15. Gould RL, Coulson MC, Patel N, Highton-Williamson E, Howard RJ. Interventions for reducing benzodiazepine use in older people: metaanalysis of randomised controlled trials. Br J Psychiatry 2014;204(2):98107.

16. Tannenbaum C, Martin P, Tamblyn R, Benedetti A, Ahmed S. Reduction of inappropriate benzodiazepine prescriptions among older adults through direct patient education: the EMPOWER cluster randomized trial. JAMA Intern Med 2014;174(6):890-898.

17. Kurko TA, Saastamoinen LK, Tahkapaa S, et al. Long-term use of benzodiazepines: Definitions, prevalence and usage patterns - a systematic review of register-based studies. Eur Psychiatry 2015;30(8):10371047.

18. Analytics TH. Micromedex® 1.0 (Healthcare Series), (electronic version). Greenwood Village, Colorado, USA.

19. Shadmi E, Balicer RD, Kinder K, Abrams C, Weiner JP. Assessing socioeconomic health care utilization inequity in Israel: impact of alternative approaches to morbidity adjustment. BMC Public Health 2011;11:609.

20. Thorell K, Ranstad K, Midlov P, Borgquist L, Halling A. Is use of fall risk-increasing drugs in an elderly population associated with an increased risk of hip fracture, after adjustment for multimorbidity level: a cohort study. BMC Geriatr 2014;14:131.

21. Lund BC, Chrischilles EA, Carter BL, Ernst ME, Perry PJ. Development of a computer algorithm for defining an active drug list using an automated pharmacy database. J Clin Epidemiol 2003;56(8):802-806. 
22. Marom O, Rennert G, Stein N, Landsman K, Pillar G. Characteristics and trends in hypnotics consumption in the largest health care system in Israel. Sleep Disord 2016;2016:8032528.

23. Bertisch SM, Herzig SJ, Winkelman JW, Buettner C. National use of prescription medications for insomnia: NHANES 1999-2010. Sleep 2014;37(2):343-349.

24. Isralowitz R, Reznik A, Borkin S. Late-life benzodiazepine use among Russian-speaking immigrants in Israel. Gerontologist 2006;46(5):677-679.

25. Winkelman JW. CLINICAL PRACTICE. Insomnia disorder. N Engl J Med 2015;373(15):1437-1444

26. Bell CM, Fischer HD, Gill SS, et al. Initiation of benzodiazepines in the elderly after hospitalization. J Gen Intern Med. 2007;22(7):1024-1029.

27. Grad R, Tamblyn R, Holbrook AM, Hurley J, Feightner J, Gayton D Risk of a new benzodiazepine prescription in relation to recent hospitalization. J Am Geriatr Soc. 1999;47(2):184-188.
28. Zisberg A, Shadmi E, Sinoff G, Gur-Yaish N, Srulovici E, Shochat T. Hospitalization as a turning point for sleep medication use in older adults: prospective cohort study. Drugs Aging. 2012;29(7):565-576.

29. Geulayov G, Lipsitz J, Sabar R, Gross R. Depression in primary care in Israel. Isr Med Assoc J. 2007;9(8):571-578.

30. Ayalon L, Karkabi K, Bleichman I, Fleischmann S, Goldfracht M. Between modern and traditional values: Informal mental health helpseeking attitudes according to Israeli Arab women, primary care patients and their providers. Int J Soc Psychiatry. 2015;61(4):386393.

31. Kaplan G, Glasser S, Murad H, et al. Depression among Arabs and Jews in Israel: a population-based study. Soc Psychiatry Psychiatr Epidemiol. 2010;45(10):931-939. 